University of Wollongong

Research Online

Faculty of Social Sciences - Papers (Archive) Faculty of Arts, Social Sciences \& Humanities

2013

The alpha band of the resting electroencephalogram under pulsed and continuous radio frequency exposures

Nick Perentos

RMIT University

Rodney Croft

University of Wollongong, rcroft@uow.edu.au

Raymond J. McKenzie

Swinburne University of Technology, Ray.McKenzie@team.telstra.com

Irena Cosic

RMIT University

Follow this and additional works at: https://ro.uow.edu.au/sspapers

Part of the Education Commons, and the Social and Behavioral Sciences Commons

Research Online is the open access institutional repository for the University of Wollongong. For further information contact the UOW Library: research-pubs@uow.edu.au 


\title{
The alpha band of the resting electroencephalogram under pulsed and continuous radio frequency exposures
}

\begin{abstract}
The effect of GSM-like electromagnetic fields with the resting electroencephalogram (EEG) alpha band activity was investigated in a double-blind cross-over experimental paradigm, testing the hypothesis that pulsed but not continuous radio frequency (RF) exposure would affect alpha activity, and the hypothesis that GSM-like pulsed low frequency fields would affect alpha. Seventy-two healthy volunteers attended a single recording session where the eyes open resting EEG activity was recorded. Four exposure intervals were presented (sham, pulsed modulated RF, continuous RF, and pulsed low frequency) in a counterbalanced order where each exposure lasted for $20 \mathrm{~min}$. Compared to sham, a suppression of the global alpha band activity was observed under the pulsed modulated RF exposure, and this did not differ from the continuous RF exposure. No effect was seen in the extremely low frequency condition. That there was an effect of pulsed RF that did not differ significantly from continuous RF exposure does not support the hypothesis that 'pulsed' RF is required to produce EEG effects. The results support the view that alpha is altered by RF electromagnetic fields, but suggest that the pulsing nature of the fields is not essential for this effect to occur.
\end{abstract}

\section{Keywords}

era2015

\section{Disciplines}

Education | Social and Behavioral Sciences

\section{Publication Details}

Perentos, N., Croft, R., McKenzie, R. J. \& Cosic, I. (2013). The alpha band of the resting electroencephalogram under pulsed and continuous radio frequency exposures. IEEE Transactions on Biomedical Engineering, 60 (6), 1702-1710. 


\title{
The Alpha Band of the Resting Electroencephalogram under Pulsed and Continuous Radiofrequency Exposures
}

\author{
N. Perentos, R. J. Croft, R. J. McKenzie, I. Cosic, Member, IEEE
}

\begin{abstract}
The effect of GSM-like electromagnetic fields with the resting electroencephalogram alpha band activity was investigated in a double blind cross-over experimental paradigm, testing the hypothesis that pulsed but not continuous radiofrequency (RF) exposure would affect alpha activity, and the hypothesis that GSM-like pulsed low frequency fields would affect alpha. Seventy two healthy volunteers attended a single recording session where the eyes open resting electroencephalogram activity was recorded. Four exposure intervals were presented (sham, pulsed modulated radiofrequency, continuous $\mathrm{RF}$, and pulsed low frequency) in a counterbalanced order where each exposure lasted for 20 minutes. Compared to sham, a suppression of the global alpha band activity was observed under the pulsed modulated RF exposure, and this did not differ from the continuous RF exposure. No effect was seen in the ELF condition. That there was an effect of pulsed RF that did not differ significantly from continuous RF exposure does not support the hypothesis that 'pulsed' RF is required to produce EEG effects. The results support the view that alpha is altered by RF electromagnetic fields, but suggest that the pulsing nature of the fields is not essential for this effect to occur.
\end{abstract}

Index Terms - brain, EEG, RF, SAR, pulsed, continuous, electromagnetic radiation effects, GSM, low-level exposure

\section{INTRODUCTION}

$\mathrm{T}$ HE exposure to radio frequency (RF) electromagnetic fields that arise from the use of wireless communication devices has led to concern regarding the possibility that negative health effects may ensue. This possibility has been examined with respect to various endpoints, one of which is the resting brain activity as measured by the

Manuscript received 15 Oct, 2012. This work was partly funded by the National Health and Medical Research Council (NHMRC).

N. Perentos was with the Department of Electrical and Electronics Engineering, RMIT University, Melbourne, Australia. He is now with the Pharmacology Department, University of Cambridge, UK. (np368@cam.ac.uk).

R. J. Croft, was with the Brain Sciences Institute, Swinburne University, Melbourne, Australia. He is now with the School of Psychology, University of Wollongong, Wollongong NSW 2522 Australia. (corresponding author email: rcroft@uow.edu.au, phone: +61 24221 3652).

R. J. McKenzie is with the Chief Technology office, Telstra Corporation, Melbourne, Australia (e-mail: ray.mckenzie@team.telstra.com).

I. Cosic is with the Science, Engineering \& Health Office, RMIT University, Melbourne, Australia (irena.cosic@rmit.edu.au) electroencephalogram (EEG). Unlike most cognitive endpoints measured, the EEG has proven to be reliably affected by low level RF such as mobile phones [1]. In terms of waking EEG, the effect of RF has predominantly been shown to increase power in the $\sim 8-12 \mathrm{~Hz}$ or alpha band [2]-[6], although some reductions of this component have also been reported [7]. In terms of sleep EEG, the $\sim 12-15 \mathrm{~Hz}$ or sleep spindle frequency range is enhanced subsequent to exposure [8]-[14], with some indication that this effect is dose-dependent [14]. Further, Loughran and colleagues recently demonstrated that the sleep EEG effect was replicable in the same individuals over time, with individual differences in response to the RF also consistent over time [12]. The magnitude of these effects have consistently been reported as small $(\sim 10 \%)$, and there is no indication that they relate to significant impairment. For example, no clear pattern of cognitive impairment has been reported during waking, nor has any clinically-significant index of sleep quality [8]-[11],[14].

International RF safety standards (e.g. IEEE C95.1 [15], ICNIRP 1998 [16]) are based heavily on an understanding of the effect of heat on safety, as the only known biophysical mechanism by which RF can affect biological tissue is through heating [17]. However, as the levels of RF used in the above studies are only able to increase temperature in the brain by a negligible amount (circa $0.1 \mathrm{C}^{\circ}$, over temporal cortex), thermal mechanisms do not intuitively appear able to explain the above EEG effects. Thus although these EEG effects do not directly correspond to health effects, they suggest a limitation in current bioelectromagnetics knowledge that is important to RF health standards and needs to be resolved.

Some research has thus attempted to determine the exposure characteristics that are relevant to the EEG changes, in an attempt to help ascertain what the mechanism of interaction is. For example, in a series of pioneering studies, the Achermann laboratory has established that sleep EEG effects occur under pulse modulated (PM) RF exposures but not continuous wave (CW) RF exposures [4],[10]. This has been argued to show that the effect is non-thermal, as the total RF energy deposited (and thus resultant temperature increase) was the same in the $\mathrm{CW}$ and PM conditions. Further, they have shown that the magnitude of sleep EEG effect is dependent on the frequency of the pulse (when Specific Absorption Rate (SAR) is matched), with $14 \mathrm{~Hz}$ resulting in a greater effect than $217 \mathrm{~Hz}$ 
pulsing [14].

However, a limitation with concluding that the effect of pulsing represents a non-thermal effect is that it does not account for differences in SAR as a function of time. That is, although when averaged over a 1-second interval there was no difference in SAR between the CW and PM RF of the above papers [4],[10],[14], this time-averaged definition of SAR obscures an important difference between the CW and PM conditions. Specifically, the instantaneous peak in situ field is different in the two conditions. For example, the $14 \mathrm{~Hz}$ pulsing of Schmidt and colleagues [14], in order to produce a timeaveraged SAR that was equivalent to the $\mathrm{CW}$ condition, had a SAR (temporal) peak of $61.9 \mathrm{~W} / \mathrm{kg}$ versus $16 \mathrm{~W} / \mathrm{kg}$ for the CW condition. Thus the PM condition had greater instantaneous energy deposition than the CW for brief, repeating periods, and so the SAR differed between the conditions as a function of time. The result of this difference is that thermal mechanisms can still be invoked as possible explanations of the phenomenon, whereby a thermal threshold may have been reached in the PM but not CW condition. If such a 'thermal threshold' model can explain the EEG results reported above, this would obviate the need to develop a new non-thermal mechanism to explain the phenomenon.

Further to the radio frequency exposures discussed above, handsets such as those used for GSM communications are also a source of extremely low frequency (ELF) pulse modulated fields [18]. Due to the difference in spectral content of the ELF fields (compared to RF) it is possible that these fields may interact differently, if at all, with the human EEG. EEG studies with exposures that share some resemblance to the GSM-ELF fields have shown changes in resting brain activity (albeit inconsistently), thereby justifying further investigation with mobile phone-like exposure conditions [19]-[21].

The aim of this study was to examine the potential impact of pulsing of the RF field on the resting EEG by comparing CW to PM RF exposures with identical instantaneous peak field levels (which results in a higher time-averaged SAR level for CW than PM). A secondary aim was to determine whether a GSM-like ELF field would also affect the resting EEG.

\section{MATERIALS AND METHODS}

\section{A. Subjects}

Seventy two healthy volunteers (35 female, 37 male) participated in the study. The mean age of the sample was 24.5 years (standard deviation $=5.4$ years). Participants were instructed to abstain from alcohol consumption for the 24-hour period prior to the experiment, and abstain from mobile phone use and caffeine consumption within the six-hour period prior to the experiment. The Human Research Ethics Committees of RMIT University and Swinburne University of Technology approved the study. Written informed consent was obtained from each participant prior to any experimental procedures. A monetary reimbursement was made available for subjects who concluded the experimental protocol.

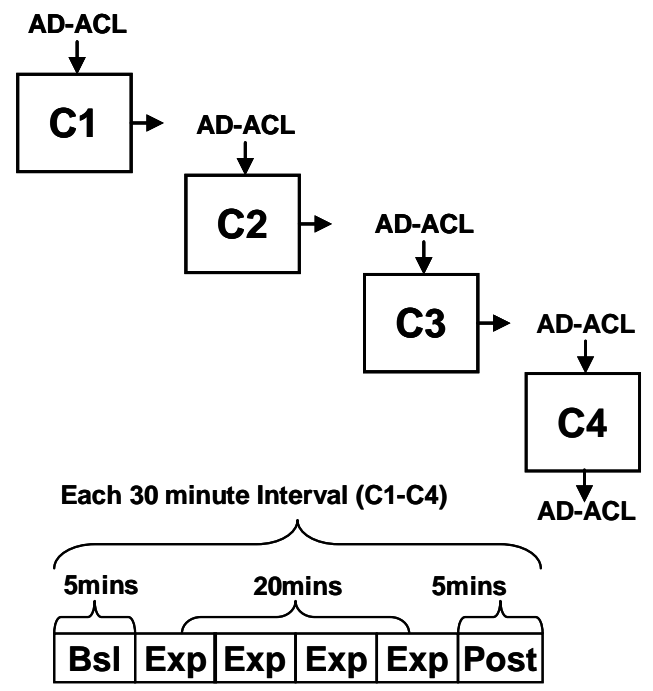

Fig. 1. The experimental protocol comprised of four time intervals (C1-C4). Exposure conditions were assigned in a counterbalanced order to each of C1-C4. Between intervals the Activation Deactivation Adjective Check List (AD-ACL) was administered. Bsl, baseline interval; Exp, exposure interval; Post, post exposure interval.

\section{B. Protocol}

Participants attended a two-hour recording session, detailed in Fig. 1, consisting of four 30-minute intervals of EEG recording. In each interval participants received one of four exposure conditions; Sham, where no electromagnetic fields were present, Continuous RF (CW RF), Pulsed RF, mimicking the Discontinuous Transmission mode (DTX) signal structure of $2^{\text {nd }}$ generation mobile phones (PM RF), and DTX pulsed low frequency (ELF) electromagnetic exposure, mimicking that generated by intermittent current flow within the handset device of $2^{\text {nd }}$ generation mobile phones. The order of exposure was fully counter-balanced and randomly assigned, using a double blind cross-over design.

Double blinding was achieved using a pre-programmed microcontroller (Renesas M16C) that controlled the RF and ELF signal generator output. A person unassociated with data collection and analysis selected one of twelve software realizations (each realization included a unique set of order of exposure sequences) and uploaded it to the microcontroller unit. The experimenter would then assign one of twenty four exposure sequences to each subject using the microcontroller. Note that the experimenter had no opportunity to determine the exposure status until the software version used was revealed (which was after the data collection and analysis). Each 30minute interval consisted of five minutes that were exposurefree (which served as a baseline), twenty minutes of the exposure condition, and then five minutes that were exposurefree. EEG data were recorded continuously throughout, while participants were seated comfortably with their eyes open. Although the characteristic alpha peak is suppressed during eyes open when compared to the eyes closed state, due to the long recording intervals the eyes open condition helps to avoid large fluctuations in alertness, and reduces the chance of an alpha ceiling effect. Four-minute breaks occurred between 
each 30-minute interval, during which participants were asked to complete the Activation Deactivation Adjective Checklist, (AD-ACL) [22] and offered the opportunity to stretch and drink water.

\section{Data Acquisition}

Each participant was fitted with a Compumedics Neuroscan 19-channel tin EEG Quick Cap, which employed the standard 10/20 international electrode positioning system, referenced to the left mastoid (M1) and grounded to the point midway between $\mathrm{FPz}$ and $\mathrm{Fz}$. In addition, the electrooculogram was recorded from above (E1) and below (E3) the left eye, and the outer canthi of the left (E5) and right (E6) eyes. Data were recorded using the Synamps ${ }^{2 \mathrm{TM}}$ system (Compumedics, Ltd) with a sampling frequency of $250 \mathrm{~Hz}$. Electrode impedances were below $5 \mathrm{k} \Omega$ at the start of recordings, which took place in an electromagnetically metal-shielded room. To determine shielding effectiveness and ambient fields within the room, a Narda SRM 3000 frequency selective radiation meter (Hauppauge, NY) was used. With reference to externally incident ambient fields, shielding effectiveness was approximately $25 \mathrm{~dB}(800 \mathrm{MHz}$ to $2.45 \mathrm{GHz})$, with the maximum measured level at the subject's head less than 30 $\mathrm{mV} / \mathrm{m}$ at any frequency within that range. Both the subject and the experimenter were present in the recording room, but no visual contact was possible either way between subject and experimenter during EEG recordings.

\section{Shielding of EEG Amplifiers}

To shield against electromagnetic interference that EEG amplifiers may be affected by, the amplifiers were placed in a specially constructed metallic box. The box was fitted with a high frequency electromagnetic interference gasket and copper tape at all joints. To prevent the currents induced on the EEG leads from entering the amplifier unit, each lead was fed into the box via a $\pi$-section filter (TUSONIX 4209-053). The output data cable was fed through a copper mesh which was then soldered to the shielding box and ferrite loaded. The selected filters achieve good current flow at low frequencies while attenuating RF currents $(900 \mathrm{MHz})$ by at least $65 \mathrm{~dB}$. Tests on a dummy head realized using a watermelon (due to high water content, a water melon serves as a rough approximation of the dielectric properties of the head, and also provides an electrical path between electrodes) demonstrated the effectiveness of the shielding method. Tests were performed at power levels $\sim 3 \mathrm{~dB}$ higher than those used during actual experiments and noise pickup was maximized by wrapping the EEG leads around the radiation source. Noise suppression is clearly demonstrated in Fig. 2.

\section{E. Electromagnetic Exposure}

\section{Handset and Positioning}

All exposures were delivered through a specially constructed handset. The handset was designed to allow for selective RF and ELF exposures while mimicking the spatial exposure characteristics that would be expected from real GSM handsets. In the RF domain this was achieved with a Planar Inverted F-type Antenna (PIFA) implemented as a modified design of that presented by Kivekas et al. [23]. For all subjects, the handset was placed according to the standard ear to mouth position, over the right hemisphere. The speaker and antenna were located over the auditory canal. The phone was held in place with a specially constructed cradle shown in Fig. 3 (also described in Boutry et al. [24]). Additionally, another handset that was not radiating, but was otherwise identical, was placed on the left side of the head so as to avoid lateralization of participants' attention.

\section{RF exposure}

The SAR performance of the handset was evaluated at an accredited commercial facility (EMC technologies, Melbourne, Australia). It was performed on the complete handset (radiating element plus casing), thus mimicking performance under real conditions of use. The standard ear to mouth positioning was used against a Standard Anthropomorphic Model (SAM) right section human head phantom, filled with tissue equivalent liquid $(\sigma=0.979801$ $\mathrm{S} / \mathrm{m}, \varepsilon_{\mathrm{r}}=42.1673 ; \rho=1000 \mathrm{~kg} / \mathrm{m}^{3}$ ). CW RF exposures were set at a 10 gram peak spatial-average SAR level of $1.95 \mathrm{~W} / \mathrm{kg}$. According to relevant standards, the maximum permissible level of exposure, averaged over any 6 minute interval, is 2 W/kg [15]-[16]. Peak RF fields during PM RF were kept equal to those of the CW RF exposure. As a result, and due to the structure of the DTX signal where most time slots are inactive (with main frequency components at 2.1, 8.3 and $217 \mathrm{~Hz}$ ), the SAR level of the PM RF exposure dropped to $0.06 \mathrm{~W} / \mathrm{kg}$. Assuming that differences between the effects produced by these two exposures are not due the different SAR levels, as

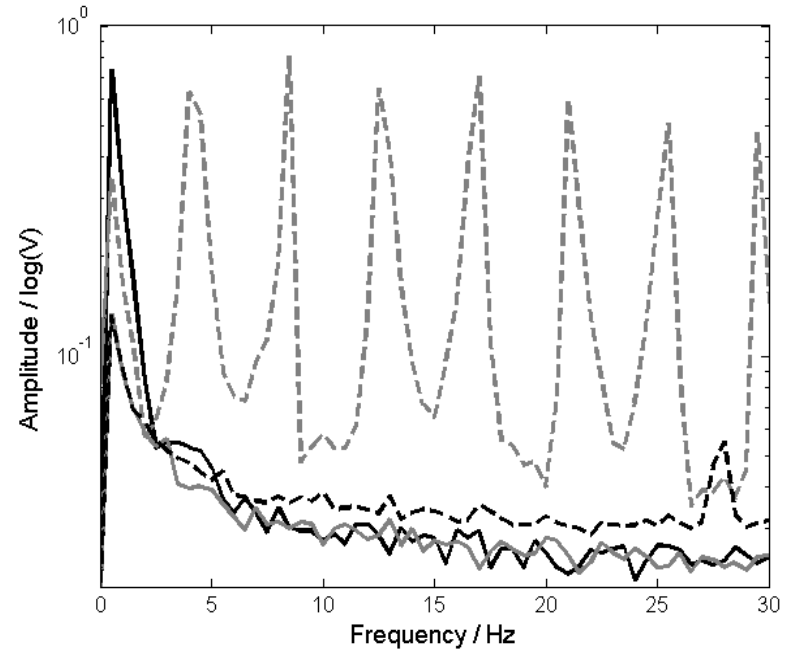

Fig. 2. Spectral output from the EEG amplifier is shown demonstrating suppression of the electromagnetic noise. Dashed grey line; pulsed RF on and unshielded amplifier, Solid black line; ELF on and amplifier shielded; Solid grey line; RF on with shielded amplifier and Dashed black line; RF and ELF off with shielded amplifier. 


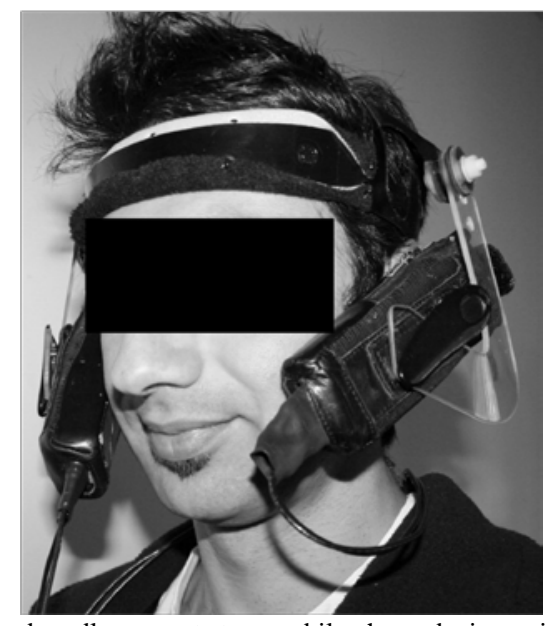

Fig. 3. The head cradle supports two mobile phone devices with the one next to the right side of the head radiating while the one on the left was inactive. The devices were otherwise identical in appearance and weight.

would be suggested by the results of Regel et al. and Huber et al. [4],[10], then this approach allows testing the importance of the pulsing by removing any instantaneous peak field differences which can lead to threshold related effects. SAR levels for both exposures are well below levels that can produce thermal effects, so any observed differences would likely be attributed to non-thermal phenomena. With instantaneous energy depositions identical and a PM RF exposure SAR well below that of continuous, any effects during PM RF exposures would have to be attributed to the pulsed nature of the exposure.

\section{ELF Exposure}

Consistent with measured values of GSM handset-generated ELF fields [18], a peak field of $25 \mu \mathrm{T}$ was generated at the front surface of the handset. The field level was verified using the measurement setup described in Perentos et al., [18]. Through modeling performed in the CST Studio Environment, exposures were shown to amount to a spatially averaged maximum of $7.77 \mu \mathrm{A} / \mathrm{m}^{2}$ equivalent to less than $1 \%$ of permissible levels. The pulsing of the ELF field was identical to that used during the PM exposure (see section "RF Exposure”).

\section{F. Data Analysis}

Data were analyzed using the EDIT 5.5.2 software (Scan 4.3, Compumedics ${ }^{\mathrm{TM}}$ ). EEG data were re-referenced to the numerical average of the left and right mastoids (channels M1 and M2 respectively) and submitted through the revised aligned-artifact average automated ocular artifact reduction routine [25]. EOG corrected data were epoched into 4-second intervals, spline-fitted to 1024 samples and the mean removed (DC correction). Epochs containing EEG data with residual voltages greater than $\pm 200 \mu \mathrm{V}$ were considered to contain artifacts and were rejected. Remaining epochs were then grouped into six 5-minute intervals (pre, during 1, during 2, during 3, during 4, and post exposure). A 1024 point Fast Fourier Transform with a $10 \%$ cosine window function was

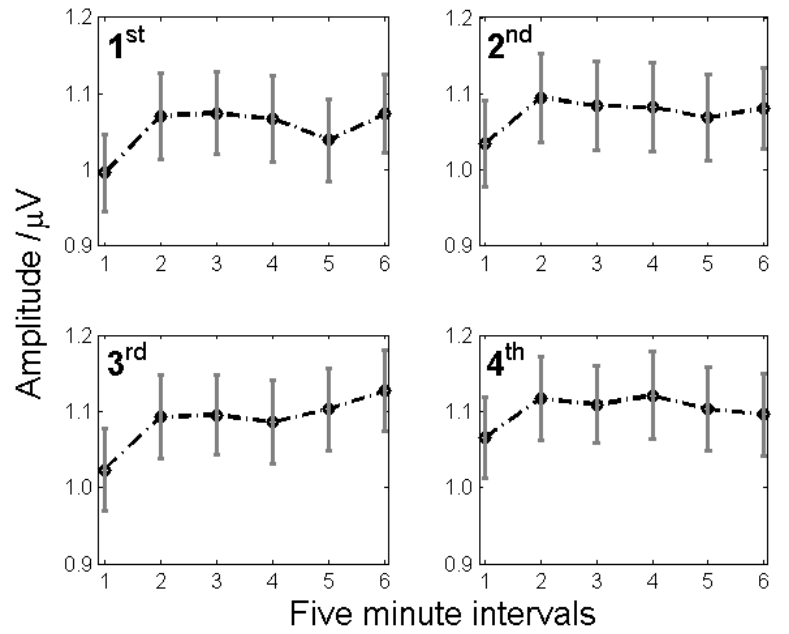

Fig. 4. The time course of the global mean alpha band activity is depicted for separate experimental intervals grouped irrespective of exposure condition. There is a tendency of alpha activity to increase within 30-minute intervals. $1^{\text {st }}, 2^{\text {nd }}, 3^{\text {rd }}$ and $4^{\text {th }}$ correspond to the first to fourth experimental interval respectively, the $\mathrm{x}$-axis represents the six, five-minute intervals that make up each thirty-minute experimental condition, and error bars depict the standard error of the mean

used to obtain amplitude frequency spectra which were subsequently averaged in the Alpha $(8-12.75 \mathrm{~Hz})$ band.

\section{G. Statistical Analysis}

The statistical analysis was performed on spectral amplitude DIFFERENCE data obtained through subtraction from the baseline spectral amplitude data (i.e. 'during exposure' minus 'baseline', or 'post exposure' minus 'baseline'). Electrodes were grouped by averaging to reduce noise and the number of statistical comparisons. Electrode groups were Left Frontal (Fp1, F3, F7), Midline Frontal (Fz), Right Frontal (Fp2, F4, F8), Left Central (C3, T7), Midline Central (Cz), Right Central (C4, T8), Left Posterior (P3, P7, O1), Midline Posterior (Pz) and Right Posterior (P4, P8, O2). To remove effects associated with the duration of experiment, data were grouped according to time intervals irrespective of exposure condition, (i.e. $1^{\text {st }}$, $2^{\text {nd }}, 3^{\text {rd }}$, and $4^{\text {th }} 30$-minute intervals), and corresponding $\mathrm{z}$ scores were calculated for each interval. As such, data of the complete sample grouped per experimental interval had identical means of 0 and standard deviations of 1 . As a large number of dependent variables could not be normalized, nonparametric statistical procedures were employed (Wilcoxon Signed Rank tests). Since this non-parametric procedure has limited flexibility, new variables were calculated to test specific a priori and exploratory hypotheses as detailed next. For each of the 'energetic arousal' and 'tense arousal' subscales of the AD-ACL questionnaire, data were converted to difference values (computed as the subscale score at the end of the half hour condition minus the subscale score at the start of the half hour condition). Resultant data were then converted to Z-scores as per the EEG data above.

\section{Hypothesis-Driven Tests}

Non-directional tests were performed on the DIFFERENCE 
values derived during exposure, to determine whether there was an effect of the exposures on global alpha activity (calculated as the mean of all scalp region alpha difference values). This resulted in two comparisons for the pulse modulation hypothesis (Sham versus PM; PM versus CW), and one for the ELF hypothesis (Sham versus ELF); no adjustments for multiple comparisons was made as analyses were $a$ priori and less than the number of treatment conditions [26].

\section{Exploratory Tests}

The hypothesis driven tests were repeated for the postexposure period. In addition, effects of laterality and sagitality were assessed (using the differences between ipsilateral and contralateral, and frontal and posterior regions respectively). To correct for multiple comparisons the Dubey/Armitage \& Parmar method (D/AP) was employed [27] for each of the Pulse Modulation and ELF hypothesis separately. This method accounts for the correlation between variables and has been used previously on EEG data [2]. For the Pulse Modulation hypothesis, there were 10 tests and a mean correlation of 0.180 , which results in an adjusted alpha level of 0.008 . For the ELF hypothesis, there were 5 tests and a mean correlation of 0.188 , which resulted in an adjusted alpha level of 0.008 . Uncorrected p-values are shown in the results section and interpreted relative to these adjusted critical alpha values.

\section{Investigation for the Presence of Carry-Over Effects}

Since a single-day protocol was employed (multiple exposures in the same day), it is important to examine whether carry-over effects have influenced results. This is especially crucial in light of reports of delayed effects on brain activity as seen in sleep [11], [14] as well as resting EEG [4],[10] studies. The examination was carried out in two ways. First is an empirical evaluation of whether the pre-exposure periods differed between condition (should there be any carry-over effects, this would be expected to be seen if exposure had an effect that lasted beyond exposure cessation). The second addressed the possibility that, if EMF effects were present, the different order of conditions may have explained such effects. That is, to achieve a fully counterbalanced protocol, the Sham condition followed the active (EMF) conditions more times than the active conditions followed the Sham, which could potentially be a source of bias if the EMF effect exceeds the 30 minute time interval. This was addressed mathematically and is presented in Appendix A.

\section{RESULTS}

Fig. 4. shows the overall tendency of the alpha activity to increase throughout each experimental interval. Note that this was accounted for in the analysis through the normalization procedure described above. The mean alpha amplitudes for each condition are summarized in Table 1, and the statistical findings are summarized in Table 2 and discussed further in the following Sections.

\section{Hypothesis-Driven Tests}

During PM RF exposure, the change in alpha band activity from baseline was significantly lower in comparison to the Sham condition $(\mathrm{p}=0.045)$, and this did not differ from CW exposure $(p=0.902)$. ELF exposure did not differ from Sham $(p=0.786)$. Raw mean spectral data for midline sites of all exposure conditions are shown in Fig. 5 where only small amplitude changes are noted.

\section{Exploratory Tests}

Post-exposure, there were no statistically significant differences for the Sham/PM, PM/CW or Sham/ELF comparisons. For both during and post-exposure intervals, there was no interaction between either Sagitality or Laterality for the Sham/PM, PM/CW or Sham/ELF comparisons ( $\mathrm{p}>$ 0.008). Analysis of the AD-ACL data did not reveal any significant associations between exposure condition and either the energy activation or tension activation subscales.

\section{Carry-Over Effects}

Assuming that there was no carry-over effect, alpha power in the pre-exposure period would be same for the different conditions. To test this assumption, alpha power in the preexposure period was converted to Z-scores (for each time period separately, as per the main analysis), and repeated measures contrasts performed comparing Condition levels (Sham versus PM, PM versus CW, Sham versus ELF). As can be seen in Fig. 6, alpha was lower in the Sham than ELF condition $(F[1,71]=12.48, p=0.001)$, did not differ between the $\mathrm{PM}$ and $\mathrm{CW}$ condition $(\mathrm{F}[1,71]=0.21, \mathrm{p}=0.648)$, and Sham was lower but at a non-significant level than the PM condition $(F[1,71]=2.43, p=0.124)$. This raises the possibility that the baseline period may have affected subsequent difference results. 
To explore this possibility further, the above within-subject Z-scores were treated as between-subject and entered into a Backward multiple linear regression, with binary independent variables (defined as whether or not the 'immediately' preceding condition was PM, CW, or ELF, and whether there was 'any' preceding variable that was PM, CW, or ELF (entry and removal criteria were $\mathrm{p}<0.05$ and $\mathrm{p}<0.10$ respectively). Satisfaction of these conditions was coded as ' 1 ', and nonsatisfaction as ' 0 '. Using a reduced degrees-of-freedom to account for the true number of participants $(\mathrm{N}=72)$, the best model was significant $(\mathrm{F}[2,69]=4.58, \mathrm{p}<0.05)$, with 'any' previous ELF $(B=-0.311)$ or CW $(B=0.236)$ exposure predicting smaller and larger normalized alpha values respectively. This suggests that ELF and CW carry-over effects may have affected pre-exposure levels.

The mathematical treatment of the inequality of exposure orders (Sham being preceded by active exposures more often than active exposures being preceded by Sham) is described in Appendix A. That demonstrated that the maximal distortion would occur when an effect of an active exposure propagates to the 'pre-exposure' interval, but not to the 'during exposure' interval of the subsequent experimental condition, has a magnitude four times greater than the main effect, and is in the opposite direction (i.e. a rebound effect). It is difficult to determine how likely such a scenario is based on the imprecision of the present carry-over effect analysis, but given

TABLE I

MEAD (SD) OF ALPHA AMPLitudes

\begin{tabular}{|c|c|c|c|c|c|c|}
\hline \multirow[b]{3}{*}{$\mathrm{Fr}$} & \multicolumn{3}{|c|}{ SHAM } & \multicolumn{3}{|c|}{ PM RF } \\
\hline & baseline & during & post & baseline & during & post \\
\hline & $\begin{array}{c}0.96 \\
(0.38)\end{array}$ & $\begin{array}{c}1.06 \\
(0.41)\end{array}$ & $\begin{array}{r}1.09 \\
(0.43)\end{array}$ & $\begin{array}{c}1.02 \\
(0.44)\end{array}$ & $\begin{array}{c}1.09 \\
(0.44)\end{array}$ & $\begin{array}{c}1.11 \\
(0.42)\end{array}$ \\
\hline Ps & $\begin{array}{c}1.04 \\
(0.48)\end{array}$ & $\begin{array}{c}1.10 \\
(0.49)\end{array}$ & $\begin{array}{r}1.12 \\
(0.52)\end{array}$ & $\begin{array}{c}1.10 \\
(0.57)\end{array}$ & $\begin{array}{c}1.12 \\
(0.54)\end{array}$ & $\begin{array}{r}1.16 \\
(0.52)\end{array}$ \\
\hline Ip & $\begin{array}{c}0.91 \\
(0.36)\end{array}$ & $\begin{array}{c}0.99 \\
(0.38)\end{array}$ & $\begin{array}{c}1.01 \\
(0.40)\end{array}$ & $\begin{array}{c}0.97 \\
(0.42)\end{array}$ & $\begin{array}{c}1.01 \\
(0.41)\end{array}$ & $\begin{array}{r}1.03 \\
(0.40)\end{array}$ \\
\hline Cn & $\begin{array}{c}0.91 \\
(0.37)\end{array}$ & $\begin{array}{c}0.98 \\
(0.38)\end{array}$ & $\begin{array}{r}1.00 \\
(0.41)\end{array}$ & $\begin{array}{c}1.03 \\
(0.46)\end{array}$ & $\begin{array}{c}1.00 \\
(0.42)\end{array}$ & $\begin{array}{r}1.02 \\
(0.40)\end{array}$ \\
\hline P-Ip & $\begin{array}{c}0.91 \\
(0.44)\end{array}$ & $\begin{array}{c}0.96 \\
(0.44)\end{array}$ & $\begin{array}{r}0.97 \\
(0.46)\end{array}$ & $\begin{array}{c}0.96 \\
(0.51)\end{array}$ & $\begin{array}{c}0.98 \\
(0.48)\end{array}$ & $\begin{array}{c}1.01 \\
(0.46)\end{array}$ \\
\hline \multirow[t]{3}{*}{ P-Cn } & $\begin{array}{r}0.95 \\
(0.45) \\
\end{array}$ & $\begin{array}{r}1.02 \\
(0.45) \\
\end{array}$ & $\begin{array}{r}1.02 \\
(0.46) \\
\end{array}$ & $\begin{array}{r}1.00 \\
(0.53) \\
\end{array}$ & $\begin{array}{c}1.03 \\
(0.49) \\
\end{array}$ & $\begin{array}{r}1.05 \\
(0.47) \\
\end{array}$ \\
\hline & \multicolumn{3}{|c|}{ CW RF } & \multicolumn{3}{|c|}{ ELF } \\
\hline & baseline & during & post & baseline & during & post \\
\hline $\mathrm{Fr}$ & $\begin{array}{c}1.00 \\
(0.39)\end{array}$ & $\begin{array}{r}1.07 \\
(0.42)\end{array}$ & $\begin{array}{c}1.05 \\
(0.38)\end{array}$ & $\begin{array}{c}0.99 \\
(0.42)\end{array}$ & $\begin{array}{c}1.08 \\
(0.43)\end{array}$ & $\begin{array}{c}1.08 \\
(0.41)\end{array}$ \\
\hline Ps & $\begin{array}{c}1.10 \\
(0.51)\end{array}$ & $\begin{array}{c}1.12 \\
(0.50)\end{array}$ & $\begin{array}{c}1.11 \\
(0.46)\end{array}$ & $\begin{array}{c}1.07 \\
(0.53)\end{array}$ & $\begin{array}{c}1.11 \\
(0.52)\end{array}$ & $\begin{array}{r}1.10 \\
(0.49)\end{array}$ \\
\hline Ip & $\begin{array}{c}0.96 \\
(0.37)\end{array}$ & $\begin{array}{c}1.00 \\
(0.39)\end{array}$ & $\begin{array}{r}0.98 \\
(0.35)\end{array}$ & $\begin{array}{c}0.94 \\
(0.40)\end{array}$ & $\begin{array}{c}1.00 \\
(0.40)\end{array}$ & $\begin{array}{r}1.00 \\
(0.38)\end{array}$ \\
\hline Cn & $\begin{array}{r}0.95 \\
(0.38)\end{array}$ & $\begin{array}{c}0.99 \\
(0.40)\end{array}$ & $\begin{array}{r}0.97 \\
(0.36)\end{array}$ & $\begin{array}{c}0.93 \\
(0.40)\end{array}$ & $\begin{array}{c}0.99 \\
(0.40)\end{array}$ & $\begin{array}{c}0.99 \\
(0.38)\end{array}$ \\
\hline P-Ip & $\begin{array}{c}0.95 \\
(0.45)\end{array}$ & $\begin{array}{c}0.98 \\
(0.45)\end{array}$ & $\begin{array}{r}0.96 \\
(0.40)\end{array}$ & $\begin{array}{c}0.93 \\
(0.47)\end{array}$ & $\begin{array}{c}0.97 \\
(0.47)\end{array}$ & $\begin{array}{r}0.96 \\
(0.43)\end{array}$ \\
\hline P-Cn & $\begin{array}{r}1.01 \\
(0.47) \\
\end{array}$ & $\begin{array}{c}1.03 \\
(0.47) \\
\end{array}$ & $\begin{array}{r}1.02 \\
(0.42)\end{array}$ & $\begin{array}{c}0.97 \\
(0.49)\end{array}$ & $\begin{array}{c}1.02 \\
(0.47) \\
\end{array}$ & $\begin{array}{r}1.02 \\
(0.45) \\
\end{array}$ \\
\hline
\end{tabular}

Fr; Frontal, Ps, Posterior, Ip; Ipsilateral, Cn; Contralateral, P-Ip; Posterior-Ipsilateral, P-Cn; Posterior Contralateral. All values in units of $\mu \mathrm{V}$.
TABLE II

SUMMARY OF STATISTICAL FINDINGS

\begin{tabular}{|c|c|c|c|c|}
\hline & & $\begin{array}{c}E ' \\
d f=71\end{array}$ & $\begin{array}{l}\mathrm{E} \times \mathrm{S}^{\prime} \\
\mathrm{df}=71\end{array}$ & $\begin{array}{l}\mathrm{E} \times \mathrm{L}^{\prime} \\
\mathrm{df}=71\end{array}$ \\
\hline \multirow{3}{*}{ 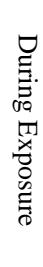 } & PM RF vs SHAM & $\begin{array}{c}p=0.045 \downarrow \\
Z=2.003\end{array}$ & $\begin{array}{l}\mathrm{p}=0.394 \\
\mathrm{Z}=0.853\end{array}$ & $\begin{array}{l}\mathrm{p}=0.207 \\
\mathrm{Z}=1.263\end{array}$ \\
\hline & PM RF vs CW RF & $\begin{array}{l}p=0.902 \\
Z=0.123\end{array}$ & $\begin{array}{l}\mathrm{p}=0.770 \\
\mathrm{Z}=0.292\end{array}$ & $\begin{array}{l}\mathrm{p}=0.783 \\
\mathrm{Z}=0.275\end{array}$ \\
\hline & ELF vs SHAM & $\begin{array}{l}p=0.372 \\
Z=0.892\end{array}$ & $\begin{array}{l}\mathrm{p}=0.480 \\
\mathrm{Z}=0.707\end{array}$ & $\begin{array}{l}\mathrm{p}=0.049 \\
\mathrm{Z}=1.970\end{array}$ \\
\hline \multirow{3}{*}{$\begin{array}{l}\text { D } \\
\mathbb{0} \\
\mathbb{1} \\
\mathbb{x} \\
0 \\
0 \\
0 \\
0 \\
0\end{array}$} & PM RF vs SHAM & $\begin{array}{l}\mathrm{p}=0.372 \\
\mathrm{Z}=0.892\end{array}$ & $\begin{array}{l}\mathrm{p}=0.054 \\
\mathrm{Z}=1.925\end{array}$ & $\begin{array}{l}\mathrm{p}=0.199 \\
\mathrm{Z}=1.285\end{array}$ \\
\hline & PM RF vs CW RF & $\begin{array}{l}\mathrm{p}=0.219 \\
\mathrm{Z}=1.229\end{array}$ & $\begin{array}{l}\mathrm{p}=0.699 \\
\mathrm{Z}=0.387\end{array}$ & $\begin{array}{l}\mathrm{p}=0.728 \\
\mathrm{Z}=0.354\end{array}$ \\
\hline & ELF vs SHAM & $\begin{array}{l}\mathrm{p}=0.203 \\
\mathrm{Z}=1.274\end{array}$ & $\begin{array}{l}\mathrm{p}=0.996 \\
\mathrm{Z}=0.006\end{array}$ & $\begin{array}{l}\mathrm{p}=0.888 \\
\mathrm{Z}=0.140\end{array}$ \\
\hline
\end{tabular}

$\mathrm{E}$; Exposure, $\mathrm{E} \times \mathrm{S}$; Exposure by Sagittality, $\mathrm{E} \times \mathrm{L}$; Exposure by Laterality. Hypothesis-driven tests are in italics ( $\mathrm{p}_{\text {crit }}=0.05$ ), and exploratory tests in normal font ( $\mathrm{p}_{\text {crit }}=0.008$ for both Pulse Modulation and ELF hypothesis)

that previous literature has failed to observe such a pattern post exposure cessation, this does not appear to be a likely explanation of the results.

\section{DISCUSSION}

The results do not support a modulation mediated effect of RF fields on the EEG. That is, although the PM RF did have an effect on alpha, this effect did not differ from the CW RF condition (where a similar alpha reduction was seen relative to Sham). To explore the lack of difference between PM RF and CW RF further, we compared CW RF to SHAM, and found no significant difference during $(Z[72]=1.560, p<0.119)$ nor post exposure $(Z[72]=1.302, p=0.193)$, although as can be seen by the during exposure p-value, there is a tendency for an effect in the same direction as the PM RF condition. Although this suggests differences between PM RF and CW RF in comparison to SHAM, this was due to the greater variability in the CW RF than PM RF condition. That is, although means were similar $(\mathrm{PM} \mathrm{RF}=-0.0794$; $\mathrm{CW}$ RF $=-0.0651$; $\mathrm{SHAM}=$ 0.1162), standard deviation was larger in the CW RF condition $(\mathrm{PM} R \mathrm{RF}=0.7070 ; \mathrm{CW} \mathrm{RF}=0.8963 ; \mathrm{SHAM}=0.7394)$. It thus may be speculated that the CW RF exposure is not as effective at eliciting changes to brain activity as is PM RF, but we have no statistical support of this supposition. Such a difference would be consistent with a threshold effect, whereby instantaneous SAR levels below this threshold may not be sufficient to affect neural function. If so, this would mean that time-averaged SAR (especially averaging over long time intervals) may not be a relevant metric for determining the effect of RF on neural function, and that peak field level needs to be taken into account. However further research is required to determine this issue. 

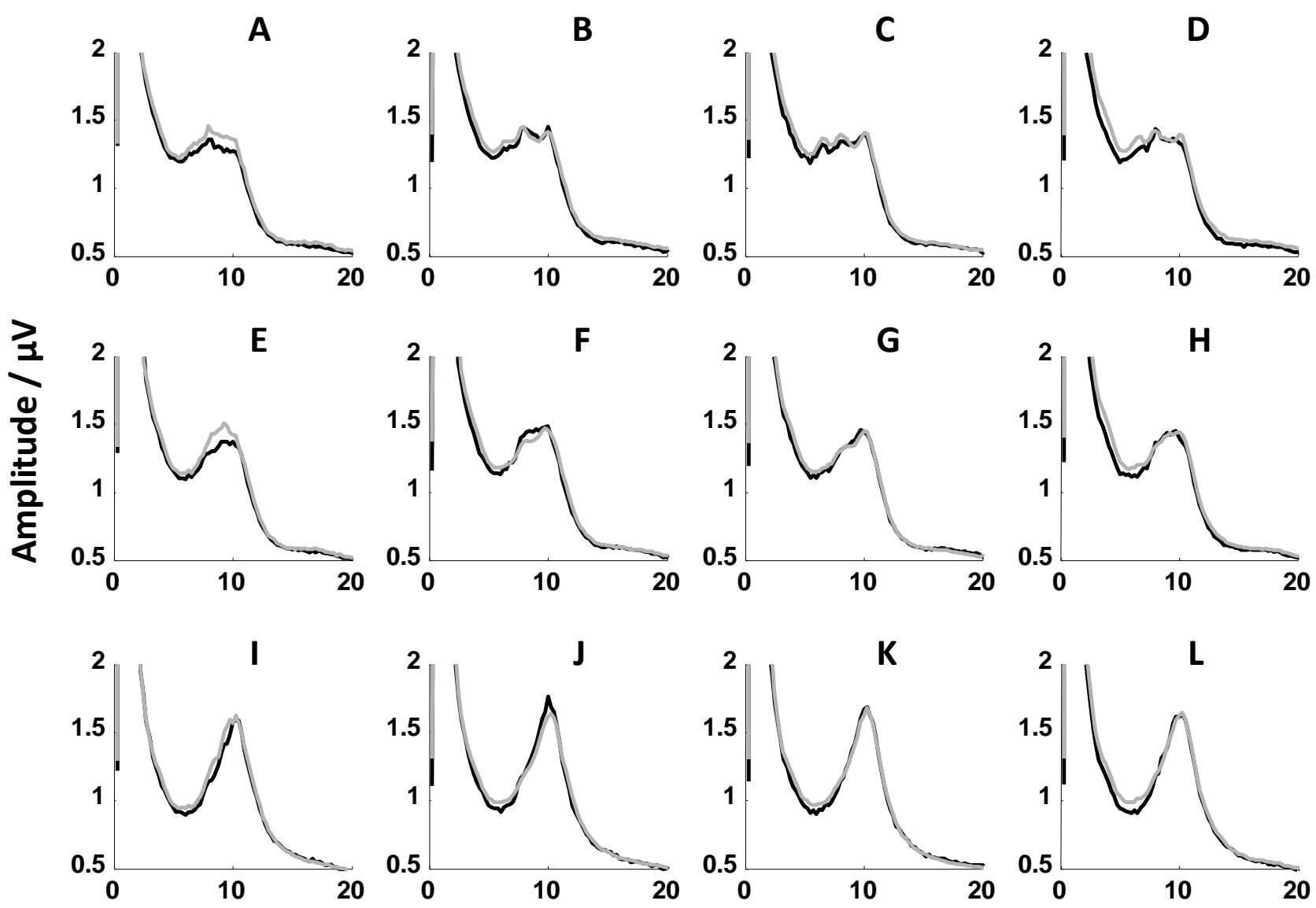

\section{Frequency / Hz}

Fig. 5. Raw amplitude spectra pre (black line) and during (grey line) exposures are shown. The $10 \mathrm{~Hz}$ alpha band peak is clearly visible and only minute changes are observed from pre to during exposure. A, E and I; Sham exposure midline frontal, midline central and midline posterior, respectively. B, F and J; PM RF exposure midline frontal, midline central and midline posterior, respectively. C, G and K; CW RF exposure midline frontal, midline central and midline posterior, respectively. D, H and L; ELF exposure midline frontal, midline central and midline posterior respectively.

It should be noted that the observed decrease in alpha is not in line with the prevailing trend of RF-induced increases in alpha activity [2]-[4],[10]. However, given that alpha changes in the literature are typically global and not localized to regions with the highest SAR, it has been suggested that the alpha change may represent an indirect effect of exposure, such as a higher level change to neural processing [28], thus leaving room for the intermediate processes to interfere with the direction of the alpha change. In addition, the current findings cannot be explained by an alpha rebound effect whereby the alpha would decrease during exposure and, consistent with literature, increase after exposure. This is the case because we did not observe a post exposure alpha increase.

This study was the first to investigate DTX-like ELF fields on EEG activity, but it did not detect any effect of this. Some relevant studies to which our results can be compared are those of rank of al [191_ron1 which employed exposures that bear nce to those employed here (pulsed I homogeneous exposure properties), 1 increases and decreases of alpha ly after exposure. In addition Stevens reported a decrease of alpha activity after exposure to weak magnetic fields [28]. Given that our results were not consistent with any of these suggests that either their results were spurious, or that the particular ELF modulation is crucial for affecting neural function. The present study cannot clarify this issue.

As a single-session protocol was used containing all four experimental conditions, it is possible that residual effects of the exposures may have contaminated the results. We investigated this possibility in two ways. Appendix A deals with a mathematical description of this problem, where it is shown that a fully counterbalanced design can still result in carry-over effects, but that a number of conditions would need to be met. Of particular relevance is that the amplitude of carry-over effect would have to be at least 4 times greater than that of the actual effect, and in the opposite direction. Although this possibility cannot be discounted, we are not aware of any empirical evidence that supports this possibility. This issue was also considered empirically, and it was found that differences occurred within the baseline periods of the conditions that would not be expected unless there were differential carry-over effects for the conditions. Specifically, 


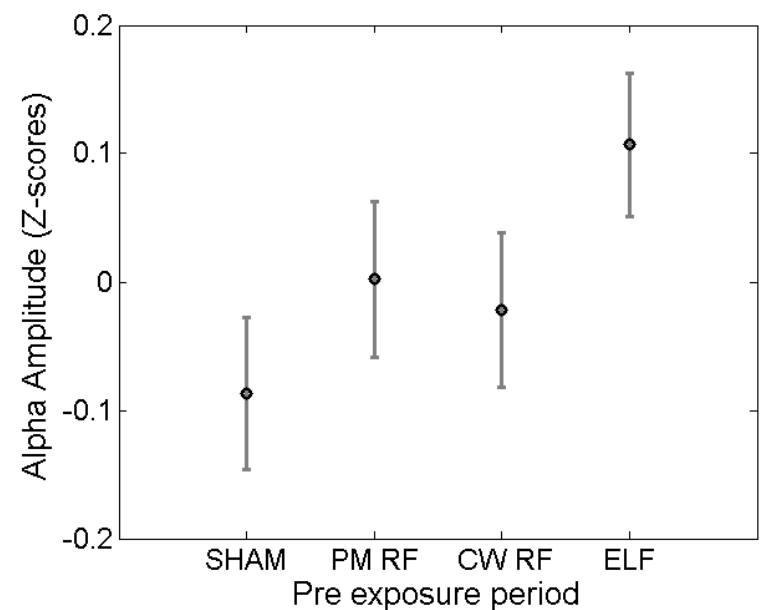

Fig. 6. Estimated marginal means (error bars show the standard error of the mean) of alpha amplitudes during the pre-exposure intervals.

following ELF exposure there was an increase in alpha amplitude and following CW exposure there was a decrease in alpha amplitude. This does not explain the present results as the pattern was similar for the exposure period itself (not reported) and thus would be substantially removed through the differencing process.

It may have confounding effects on the results in that alpha may have been differentially shifted closer to ceiling or floor effects. However, these possibilities are unlikely for the following reasons. Ceiling effects are very unlikely as we chose an eyes open condition to reduce alpha (this approximately halves alpha and leaves plenty of opportunity to see an increase), and the effect size reported in the literature due to PM RF is only circa 5-15\%. Floor effects will also not explain the results as we found a reduction (and thus no floor was demonstrated). Although having only one session reduces error variance which may otherwise occur with multiple testing sessions, we believe that the confound demonstrated in the present study present limitations for the use of single-session studies.

In summary, the current study identified changes in the resting EEG that were associated with exposures to PM RF, and that these did not differ from CW RF once instantaneous peak SAR was controlled for. The direction of this change was not consistent with the majority of the literature, but is consistent with the view that the RF effect on the EEG is an indirect response to exposure rather than being a direct result of it. No changes were seen in the DTX-like ELF exposure.

\section{APPENDIX}

To assess the amplitude of the potential carry-over effect required so as to produce the observed alpha band changes (i.e. an increase of approximately 1 more unit in the Sham relative to an Active condition) given the assumption that exposure increases alpha, mathematical calculations were performed. For this, it was assumed that all active exposures produce effects of equal magnitude and that all active exposures contribute equal carry-over effects onto the baseline of the Sham and Active conditions. For ease of interpretation we consider the absolute alpha power values, rather than the $\mathrm{z}$ scores:

1) Given that there were 4 conditions (Sham plus 3 Active), complete counterbalancing of these conditions results in 24 possible sequences of the 4 conditions. Of these, the Sham will be immediately preceded by an Active condition in 18 of its 24 occurrences ( $\mathrm{p}=0.75$ ), whereas for each Active condition only 12 of the 24 conditions will be preceded by an Active condition ( $\mathrm{p}=0.50$ ).

2) As described in the methods section, the effect (E) of a condition is defined as the difference between alpha power in the pre-exposure baseline (B) period and the immediately proceeding exposure period $(\mathrm{P})$. That is;

$$
\begin{aligned}
& E_{A}=P_{A}-B_{A} \\
& \text { and } \\
& E_{S}=P_{S}-B_{S}
\end{aligned}
$$

where the subscript ' $A$ ' is Active and ' $S$ ' is Sham.

3) As there is not an Active exposure during the baseline, it follows that the only alpha activity related to an active exposure would come from a carry-over effect, which can be represented as the proportion $\left(r_{1}\right)$ of the effect of a previous active exposure effect $\left(E_{\text {prev }}\right)$ that is still present during the baseline period. Further, given that such a carry-over effect would only occur where a condition followed an Active condition, this means that the carryover effect would occur $50 \%$ and $75 \%$ of the time for Active and Sham conditions respectively (see Point 1). Thus;

$$
\begin{aligned}
& B_{A}=0.50 r_{1} E_{\text {prev }} \\
& \text { and } \\
& B_{S}=0.75 r_{1} E_{\text {prev }}
\end{aligned}
$$

4) As there is an exposure (Sham or Active) during the period immediately proceeding the baseline period, it follows that the alpha activity related to an exposure during $\mathrm{P}$ would come from both the residual carry-over effect from the baseline, and the effect of the current exposure condition itself (note that the effect of the current exposure itself in $\mathrm{P}$ is zero for Sham). This residual carry-over effect can be represented as the proportion $\left(r_{2}\right)$ of the effect of a previous Active exposure effect that was present in the baseline period $\left(B_{A}\right)$, that was still present in $P$. That is;

$$
\begin{aligned}
& P_{A}=E+0.50 r_{1} r_{2} E_{\text {prev }} \\
& \text { and } \\
& P_{S}=0+0.75 r_{1} r_{2} E_{\text {prev }}
\end{aligned}
$$


5) Substituting (3-6) into (1) and (2), we derive the distinct effects of Active and Sham exposures as;

$$
\begin{aligned}
& E_{A}=E+0.5 r_{1} r_{2} E_{\text {prev }}-0.50 r_{1} E_{\text {prev }} \\
& \text { and } \\
& E_{S}=0+0.75 r_{1} r_{2} E_{\text {prev }}-0.75 r_{1} E_{\text {prev }}
\end{aligned}
$$

6) The difference between the Active and Sham effects, or the result (R) given in the current paper is thus equal to; $R=E+0.50 r_{1} r_{2} E_{\text {prev }}-0.50 r_{1} E_{\text {prev }}-\left(0+0.75 r_{1} r_{2} E_{\text {prev }}-0.75 r_{1} E_{\text {prev }}\right)$ $\Rightarrow R=E-r_{1} E_{\text {prev }}\left(r_{2}-1\right) / 4$

7) It can be seen from (9) that the difference (R) between the Sham and Active exposure conditions is dependent on the effect of the Exposure (E) in the current condition, the proportion $\left(r_{1}\right)$ of $E_{\text {prev }}$ from the preceding Exposure that is still present in the baseline, and the proportion $\left(\mathrm{r}_{2}\right)$ of the carry-over effect still present in the baseline, that is still present during $\mathrm{P}$.

8) It can also be seen from (9) that as $r_{2}$ gets larger (approaches 1, or $100 \%$ of the Baseline carry-over effect that is still present in the exposure condition), that $\mathrm{R}$ approaches E, whereas the maximal deviation due to this carry-over effect occurs where there is no such carry-over (i.e. the carry-over affects $B$ but not $P$, or $r_{2}=0$ ). Thus, taking the maximally-distorting carry-over effect scenario, we replace $\mathrm{r}_{2}$ with 0 , resulting in;

$R=E+r_{1} E_{\text {prev }} / 4$

9) It can be seen from (10) that if there is no carry-over effect (i.e. $r_{1}=0$ ), then the difference between the Sham and Active exposure will equal $\mathrm{E}$, the effect of the Active exposure. In order for the counterbalancing to result in the negative R from the present study (i.e. the Sham had about 1 more unit of increase from the Baseline to Proceeding period, relative to the Active condition), we can see from (10) that $r_{1}$ would need to be negative (i.e. a rebound effect rather than merely a residual affect), and it would need to be four times larger than the Active exposure effect (E) itself. For example, if $E$ was ' 1 ' ( $E_{\text {prev }}$ would also be equal to unity based on the assumption that all active exposures have the same effect magnitude), then $r_{1}$ would have to be larger than ' -4 '.

10) Different scenarios would result in different distortions to a true effect an Active exposure. From (10) we can see that the larger the rebound effect is (beyond four times the Active exposure condition itself), and the larger the Active exposure condition itself (given the above rebound effect), the more negative it would make R. As an example, if there were Active exposure and rebound effects of 1 and 5 respectively, then this would result in the observed $R$ of -0.25 , whereas values of 4 and 5 would result in the observed $\mathrm{R}$ of -1 .

11) Thus, given the maximally distorting scenario whereby there was an alpha power increase due to an Active exposure, that this resulted in a rebound effect that was present in the subsequent Baseline period and was at least four times the magnitude of that increase in alpha, and that the rebound effect has dissipated by the subsequent exposure condition, then the observed $\mathrm{R}$ in the present study of approximately -1 , could have occurred as a result of an Active exposure-related increase in alpha.

\section{ACKNOWLEDGMENTS}

The authors thank Steve Iskra for contributions in the study design, EMC Technologies for SAR assessment of the exposure device, Sarah Loughran and Arnulfo Diaz for useful discussions.

\section{REFERENCES}

[1] E. van Rongen et al., "Effects of Radiofrequency Electromagnetic Fields on the Human Nervous System", Journal of Toxicology and Environmental Health, Part B, vol. 12, pp. 572-597, Oct. 2009.

[2] R. J. Croft et al., "The effect of mobile phone electromagnetic fields on the alpha rhythm of human electroencephalogram", Bioelectromagnetics, vol. 29(1), pp. 1-10, Jan. 2008.

[3] G. Curcio et al., "Is the brain influenced by a phone call? An EEG study of resting wakefulness”, Neurosci Res, vol. 53(3), pp. 265-70, Aug. 2005.

[4] S. J. Regel et al., "Pulsed radio frequency radiation affects cognitive performance and the waking electroencephalogram", Neuroreport, vol. 18(8), pp. 803-7, May 2007.

[5] R. J. Croft et al., "Acute Mobile Phone Operation Affects Neural Function in Humans”, Clin Neurophysiol, vol. 113(10), pp. 1623-32, Oct. 2002.

[6] R. J. Croft et al., "Effects of 2G and 3G Mobile Phones on Human Alpha Rhythms: Resting EEG in Adolescents, Young Adults and Older Adults”, Bioelectromagnetics, vol. 31(6), pp. 434-445, Sep. 2010.

[7] H. D'Costa et al., "Human brain wave activity during exposure to radiofrequency field emissions from mobile phones”, Australas Phys Eng Sci Med, vol. 26(4), pp. 162-7, Dec. 2003.

[8] A. A. Borbely et al., "Pulsed high-frequency electromagnetic field affects human sleep and sleep electroencephalogram”, Neurosci Lett, vol. 275(3), pp 207-10, Nov. 1999.

[9] R. Huber et al., "Exposure to pulsed high-frequency electromagnetic field during waking affects human sleep EEG", Neuroreport, vol. 11(15), pp. 3321-5, Oct. 2000.

[10] R. Huber et al., "Electromagnetic fields, such as those from mobile phones, alter regional cerebral blood flow and sleep and waking EEG", J Sleep Res, vol. 11(4), pp. 289-295, Dec. 2002.

[11] S. P. Loughran et al., "The effect of electromagnetic fields emitted by mobile phones on human sleep”, Neuroreport, vol. 16(17), pp. 1973-6, Nov. 2005.

[12] S. P. Loughran, R. J. McKenzie, M. L. Jackson, M. E. Howard, R. J. Croft, "Individual Differences in the Effects of Mobile Phone Exposure on Human Sleep: Rethinking the Problem”, Bioelectromagnetics, vol. 33(1), pp. 86-93, Jan. 2012.

[13] M. R. Schmid et al., "Sleep EEG alterations: effects of different pulsemodulated radio frequency electromagnetic fields”, J Sleep Res., Apr. 2011., doi: 10.1111/j.1365-2869.2011.00918.x.

[14] S. J. Regel et al., "Pulsed radio-frequency electromagnetic fields: dosedependent effects on sleep, the sleep EEG and cognitive performance", J Sleep Res, vol. 16(17), pp. 253-8, Sep. 2007.

[15] IEEE Standard for safety levels with respect to human exposure to radio frequency electromagnetic fields, $3 \mathrm{kHz}$ to $300 \mathrm{GHz}$, IEEE Std 
C95.1, The Institute of Electrical and Electronic Engineers, New York USA.

[16] ICNIRP, "Guidelines for Limiting Exposure to Time-Varying Electric, Magnetic, and Electromagnetic Fields (up to $300 \mathrm{GHz}$ )", Health Phys., vol. 74 (4), pp 494-522, Apr. 1998.

[17] ICNIRP, "Review of the scientific evidence on dosimetry, biological effects, epidemiological observations and health consequences concerning exposure to high frequency electromagnetic fields $(100 \mathrm{kHz}$ to 300GHz)", P. Vecchia, R. Matthes, G. Ziegelberger, J Lin, R Saunders, A. Swerdlow (editors), ISBN 978-3-934994-10-2, 2009

[18] N. Perentos et al., "Simulation of pulsed ELF magnetic fields generated by GSM mobile phone handsets for human electromagnetic bioeffects research”, Australas Phys Eng Sci Med, vol. 31(3), pp. 235-42, Sep. 2008.

[19] C. M. Cook, A. W. Thomas, F. S. Prato, "Changes in human EEG alpha activity following exposure to two different pulsed magnetic field sequences”, Bioelectromagnetics, vol. 30(1), pp. 9-20, Jan. 2009.

[20] C. M. Cook et al., "Resting EEG effects during exposure to a pulsed ELF magnetic field”, Bioelectromagnetics, vol. 26(5), pp. 367-76, Jul. 2005.

[21] C. M. Cook, A. W. Thomas, F. S. Prato, "Human electrophysiological and cognitive effects of exposure to ELF magnetic and ELF modulated RF and microwave fields: a review of recent studies", Bioelectromagnetics, vol. 23(2), pp. 144-57, Feb. 2002.

[22] Thayer, R.E., The biopsychology of mood and arousal. 1991, New York: Oxford University Press.

[23] O. Kivekas, et al., "Bandwidth, SAR, and Efficiency of Internal Mobile Phone Antennas”, IEEE Trans. Electromagn. Compat, vol. 46(1), pp. 71-86, Feb. 2004.

[24] C. M. Boutry, et al., "Dosimetric evaluation and comparison of different RF exposure apparatuses used in human volunteer studies", Bioelectromagnetics, vol. 29(1), pp. 11-9, Jan. 2008.

[25] R. J. Croft, R. J. Barry, "EOG correction of blinks with saccade coefficients: a test and revision of the aligned-artefact average solution", Clin Neurophysiol, vol. 111(3), pp. 444-51, Mar. 2000.

[26] B. G. Tabachnick, L. S. Fidell, Using multivariate statistics, Harper \& Row (New York), 1989

[27] A. J. Sankoh, M. F. Hugue, S. D. Dubey, "Some comments on frequently used multiple endpoint adjustment methods in clinical trials", Stat Med, vol. 16(22), pp. 2529-42, Nov. 1997

[28] P. Stevens, "Affective response to 5 microT ELF magnetic field-induced physiological changes”, Bioelectromagnetics, vol. 28(2), pp. 109-14, Feb. 2007. 